\title{
Evaluation of anaphylactic reaction as an adverse event for perindopril erbumine tablets
}

\author{
*Kiran Krishnan, Kathiresan Krishnasamy \\ Department of Pharmacy, Annamalai University, Chidambaram, Tamil Nadu, India
}

\begin{abstract}
Perindopril erbumine is a tert-butylamine salt of perindopril used in the treatment of stable coronary artery disease and hypertension. The present study was aimed to evaluate the anaphylactic reaction as an adverse event for perindopril erbumine tablets. Electronic extraction of data from the safety database for this report includes all cases of anaphylactic reaction where perindopril was a primary or co-suspect drug. The study result have shown that there were overall 205 case reports for perindopril in the database, of which 200 cases were assessed as (potentially) perindopril and 05 were assessed as non-perindopril product. From the 200 case reports, 141 were serious and 59 were non-serious. Out of the 141 serious cases, 137 cases were medically confirmed while remaining 04 cases were consumer reports. Of the total 205 case reports there were five cases wherein the patient experienced anaphylactic reaction. The study concludes that three out of five cases entered in the drug safety database suggestive of a possible association between perindopril and anaphylactic reaction.
\end{abstract}

Key Words: Angiotensin-converting enzyme, coronary artery disease, hypertension, perindoprilat, system organ class, tertbutylamine salt.

\section{INTRODUCTION}

Perindopril erbumine (Tert-butylamine salt of perindopril) is a white crystalline powder, which is freely soluble in water, alcohol and chloroform. Chemically,

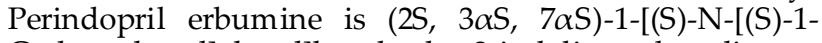
Carboxy-butyl]alanyl]hexahydro-2-indolinecarboxylic acid, 1-ethyl ester, compound with tert-butylamine (1:1). The empirical formula of Perindopril erbumine is $\mathrm{C}_{19} \mathrm{H}_{32} \mathrm{~N}_{2} \mathrm{O}_{5} \mathrm{C}_{4} \mathrm{H}_{11} \mathrm{~N}$ (figure 1) with a formula weight of 368.47 and 441.61 for the free acid and for the salt form, respectively. Perindopril is a pro-drug and it is the free acid form of perindopril erbumine (Jain et al., 2012; Sharma et al., 2011; Tarkase et al., 2013; Joseph et al., 2011). Perindopril get metabolized by hydrolysis of the ester group to form a biologically active metabolite perindoprilat, which lowers blood pressure by inhibiting the angiotensin-converting enzyme activity. Angiotensinconverting enzyme catalyze the conversion of angiotensin I to angiotensin II, which is a potent peripheral vasoconstrictor that stimulate aldosterone secretion by the adrenal cortex and provides a negative feedback on renin secretion (Riyaz et al., 2012; Sridevi et al., 2012; Varma et al., 2013; Prajapati et al., 2011). Inhibition of angiotensinconverting enzyme results in decreased plasma angiotensin II, leading to decreased vasoconstriction, increased plasma renin activity and decreased aldosterone secretion, which results in diuresis and natriuresis and may be associated with a small increase of serum potassium (Ratnaparkhi, 2012; Patil et al., 2013; Nagalakshmi et al., 2012). Perindopril erbumine tablet is indicated for the treatment stable coronary artery disease and hypertension. Perindopril has been evaluated for safety in the EUROPA (European trial on reduction of cardiac events with perindopril in stable coronary artery disease) trial,

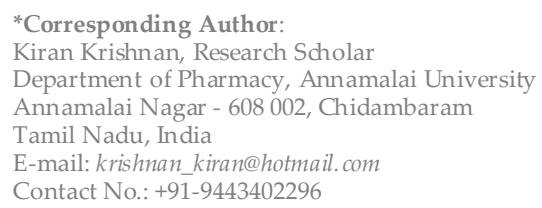

which was a multicenter, international, randomized, double blind, placebo-controlled clinical trial lasting 4 years. Twelve thousand two hundred and eighteen (12218) patients aged over 18 were randomized to $8 \mathrm{mg}$ perindopril tert-butylamine (equivalent to $10 \mathrm{mg}$ perindopril arginine $)(n=6110)$ or placebo $(n=6108)$. Hypotension was observed in 6 patients, angioedema was observed in 3 patients and sudden cardiac arrest was observed in 1 patient. However, more patients withdrew from the study for cough, hypotension or other intolerance on perindopril than on placebo (Cheng et al., 2012).

Adverse reactions that have been identified during post-approval use of Perindopril erbumine includes body pain, chills, cough, difficulty breathing, ear con gestion, fever, headache, loss of voice, nasal congestion, runny nose, sneezing, sore throat, unusual tiredness, abdominal or stomach pain, bladder pain, bloody or cloudy urine, change in hearing, chest pain, cold or flu-like symptoms, painful urination, dryness of the throat, earache, ear drainage, frequent urge to urinate, lower back pain, swollen glands, trouble with swallowing, vomiting, blurred vision, confusion, decreased urination, dizziness, faintness, irregular heartbeat, muscle cramps or pain, numbness, tingling, rapid breathing, seizures, sunken eyes, sweating, thirst, trembling, and heaviness of the legs. However, there were no preclinical data suggesting the risk or association of anaphylaxis with perindopril use. Hence, the present study was aimed to evaluation of anaphylactic reaction as an adverse event for perindopril erbumine tablets.

\section{METHODS}

As per MedDRA classification anaphylactic reaction is a "Preferred Term (PT)", which comes under the "System Organ Class" (SOC) of Immune System Disorders and the "High Level Group Term (HLGT)" Allergic Conditions. Other events such as anaphylactoid reaction, hypersensitivity, allergy and angioedema, although have different MedDRA PTs, are also under allergic conditions HLGT. Some of their symptoms overlap and sometimes they 


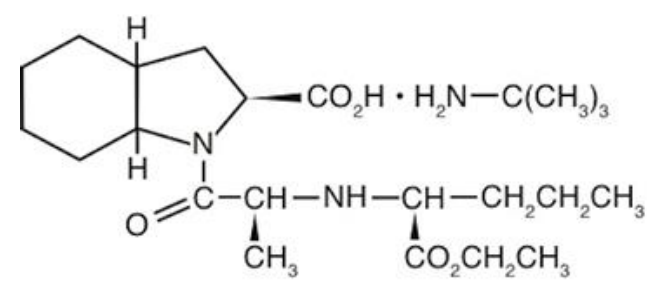

Figure 1: Chemical structure of perindopril erbumine.

might be used interchangeably by reporters of adverse drug events (ADEs). All Serious Adverse Events (SAE) from clinical trials for Perindopril (irrespective of reporter causality assessment) and all spontaneous reports of adverse events from countries where marketing authorization holder's perindipril is registered are coded onto the firm's safety database (AERS). The electronic extraction of data from the safety database for this report includes all cases of anaphylactic reaction where perindopril was a primary or co-suspect drug. A search of firms global safety database (AERS) was carried out to retrieve all cases entered in the database up to 04-Feb-2013 where perindopril was reported as primary or co-suspect medication, and a PT Anaphylactic reaction was coded as event. The retrieved data for anaphylactic reaction was analyzed under the following headings demographic, indication, report source, report type, drug dose, dechallenge/re-challenge, medical history/concurrent conditions, risk factors and concomitant medications.

\section{Patient exposure}

Perindopril has a defined daily dose (DDD) of $4 \mathrm{mg}$. From the sales data and the DDD the total number of patient days can be estimated. In total, there were about 513.93 million patient days of exposure for perindopril from Jul2006 to Jan-2013. However, this figure is of marginal relevance since it is based on worldwide sales figures of the MAH, while multiple companies market perindopril.

\section{RESULTS}

This section includes an overview of all case reports and case reports in which an anaphylactic reaction was reported. There were overall 205 case reports for perindopril in AERS database (204 reports were received during the period of 24-Jul-2006 to 4-Feb-2012), of which 200 cases were assessed as (potentially) perindopril and 05 were assessed as non-perindopril product. From the 200 case reports, 141 were serious and 59 were nonserious. Out of the 141 serious cases, 137 cases were medically confirmed while remaining 04 cases were consumer/non-health professional reports. Of the total 205 case reports there were five (05) cases wherein the patient experienced anaphylactic reaction. Graphical display of the overall case reports were displayed in figure 2.

\section{Cases presentation}

A total of five case reports were retrieved from AERS database wherein the patient experienced anaphylactic reaction. Reference number 1215 is a case report received from the Medicines and Healthcare products Regulatory Agency (MHRA), pertaining to a 78-year-old female patient who experienced angioedema and anaphylaxis while on perindopril. The patient's medical history includes anaphylaxis and angioedema with ramipril.
Concomitant medications included allopurinol, atenolol, doxazosin and gemfibrozil. The patient started taking perindopril $4 \mathrm{mg}$ daily orally and on the same day experienced angioedema and anaphylaxis. The medication was discontinued and the events were reported as resolving at the time of the report.

Reference number 0302 is a case report received from the MHRA, pertaining to an 83-year-old female patient who experienced anaphylactic reaction while using expired perindopril erbumine, bendroflumethiazide, and folic acid. The patient ran out of the current supply of medications and took some medications that she had at home which were possibly about two years old. She experienced facial and lip swelling, and presented to the Accident \& Emergency department (AED). From there the patient was admitted to hospital where she received adrenaline infusion \& nebulizers, intravenous (IV) hydrocortisone, and IV chlorphenamine. It was not certain which medications were taken (medication list was obtained from the retail pharmacy for the last 3.5yrs, and compared to those currently prescribed). Medical history of the patient includes hypertension, anaphylaxis to doxycycline and penicillin allergy (severity of reaction). Concomitant medications included amlodipine, aspirin, Calceos (calcium carbonate, colecalciferol), lansoprazole, paracetamol, simvastatin, thiamine (Vitamin B) and Vitamin B compound strong (nicotinamide, pyridoxine, riboflavin and thiamine hydrochloride).

Reference number 1044 is a case report received from the MHRA pertaining to a 75-year-old female patient who experienced anaphylaxis while using perindopril erbumine. The patient's medical history includes hypertension. Concomitant medications included oral perindopril 4mg (from 04-Mar-2010 to 12-Apr-2010) for hypertension. On 12-Apr-2010, the patient was started on perindopril erbumine $8 \mathrm{mg}$. On 24-Apr-2010, she developed anaphylaxis. On the same day, therapy with perindopril erbumine was permanently withdrawn and the patient was given an unknown treatment. The patient was advised to avoid ACE inhibitors. Anaphylaxis was considered to be life threatening and this event was resolving at the time of the report.

Reference number 2269 is a case report received from the MHRA pertaining to a 78-year-old female patient who experienced anaphylactic reaction while receiving perindopril erbumine. Medical history includes hypertension and drug allergy (multiple allergies to medication under investigation). Concomitant medications included amlodipine and levothyroxine. The patient was started on perindopril erbumine at a dose of $8 \mathrm{mg}$ orally for hypertension and developed tongue swelling suggesting an anaphylactic reaction. The drug was discontinued and the event resolved on the same day.

Reference number 1029 is a literature case report pertaining to a 73-year-old- Caucasian-female patient who developed anaphylaxis, vascular occlusion of the right middle cerebral artery, acute ischaemic stroke, drug interaction, tachypnoea, respiratory distress, left-sided hemiparesis, hemisensory loss and small haemorrhage in the right internal capsule while receiving perindopril and alteplase. The medical history of the patient includes atrial fibrillation and hypertension. Concomitant medication of the patient included aspirin as a prophylaxis for atrial fibrillation. The patient was on perindopril for hypertension. She was hospitalized with the clinical features of a right middle cerebral artery infarction corresponding to a National Institutes of Health Stroke Score (NIHSS) of 24. $\mathrm{CT}$ scan of the brain revealed hyperden se right middle 


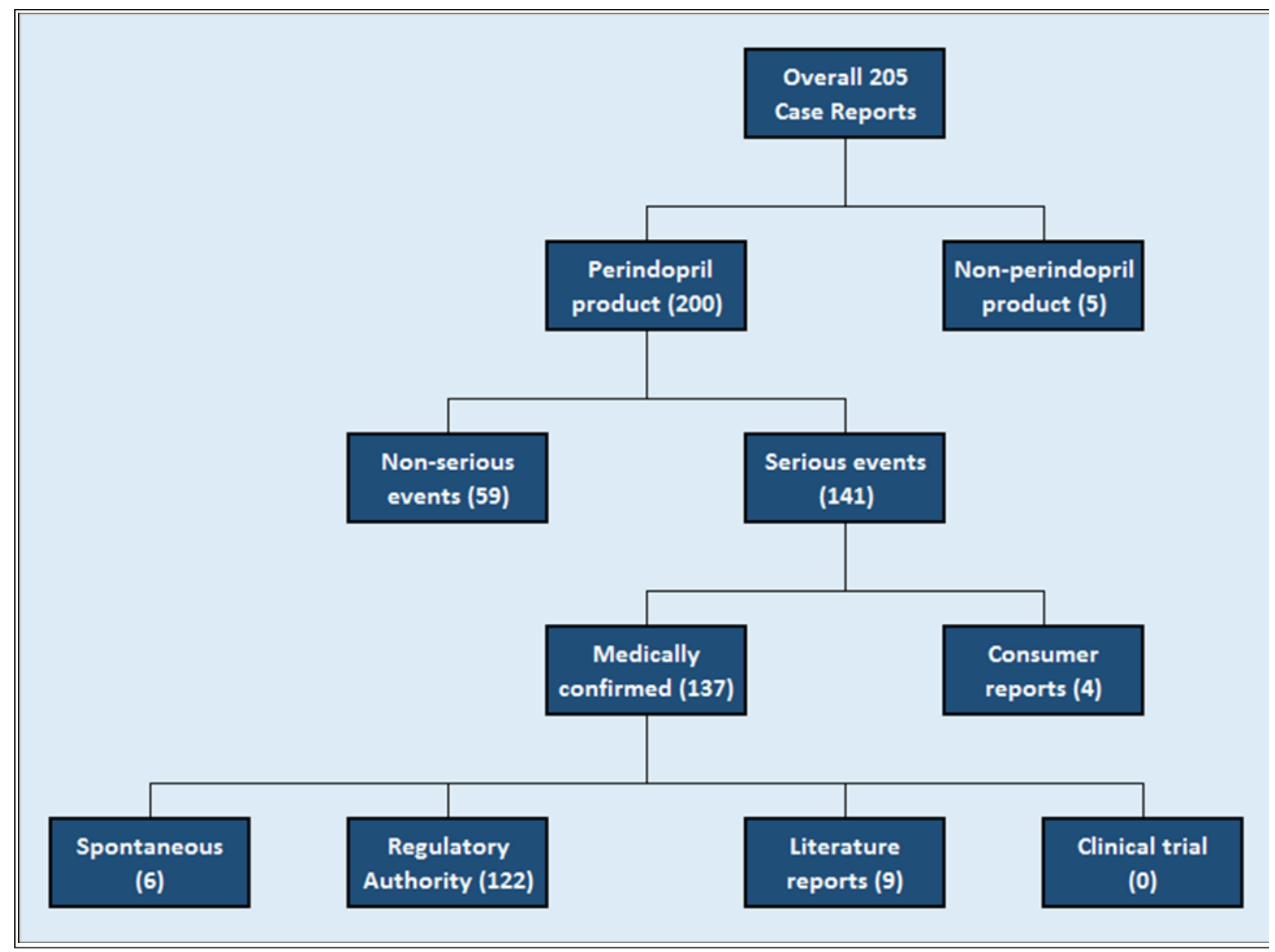

Figure 2: Graphical display of the case reports.

Table 1: Individual presentation of case reports.

\begin{tabular}{|c|c|c|c|c|c|c|}
\hline $\begin{array}{l}\text { Case } \\
\text { ID }\end{array}$ & $\begin{array}{l}\text { Age \& } \\
\text { Gender }\end{array}$ & Suspected drugs & $\begin{array}{l}\text { Latency } \\
\text { Period }\end{array}$ & SAEs (Preferred terms) & Case outcome & $\mathrm{DC} / \mathrm{RC}$ \\
\hline 1215 & $78 / \mathrm{F}$ & Perindopril & Same day & $\begin{array}{c}\text { Anaphylactic Reaction } \\
\text { Angioedema }\end{array}$ & $\begin{array}{l}\text { Recovering/ } \\
\text { Resolving }\end{array}$ & N/A \\
\hline 0302 & $83 / F$ & $\begin{array}{l}\text { Perindopril, Folic acid } \\
\text { Bendroflumethiazide }\end{array}$ & Same day & $\begin{array}{l}\text { Anaphylactic reaction } \\
\text { Intentional drug misuse }\end{array}$ & $\begin{array}{l}\text { Recovering/ } \\
\text { Resolving }\end{array}$ & N/A \\
\hline 1044 & $75 / F$ & Perindopril & 12 days & Anaphylactic reaction & $\begin{array}{l}\text { Recovering/ } \\
\text { Resolving }\end{array}$ & N/A \\
\hline 2269 & $78 / \mathrm{F}$ & Perindopril & Unknown & Anaphylactic reaction & $\begin{array}{l}\text { Recovering/ } \\
\text { Resolving }\end{array}$ & N/A \\
\hline 1029 & $73 / \mathrm{F}$ & $\begin{array}{l}\text { Perindopril } \\
\text { Alteplase }\end{array}$ & $\begin{array}{l}\text { Hours after } \\
\text { alteplase bolus }\end{array}$ & $\begin{array}{c}\text { Anaphylactic reaction, Tachypnoea, Respiratory } \\
\text { distress, Cerebral haemorrhage, Ischaemic stroke, } \\
\text { Cerebral artery occlusion, Hemiparesis Sensory } \\
\text { loss, Drug interaction }\end{array}$ & $\begin{array}{l}\text { Recovering/ } \\
\text { Resolving }\end{array}$ & N/A \\
\hline
\end{tabular}

DR/RC: De-challenge/ Re-challenge

Table 2: Statistical analysis of anaphylactic reaction for the drug perindopril.

\begin{tabular}{|c|c|}
\hline $\begin{array}{lc} & \text { Parameters } \\
\end{array}$ & Data \\
\hline Number of individual cases with the suspect medicinal product $P$ involving an adverse event (A) & 6 \\
\hline Number of individual cases with the suspect medicinal product $\mathrm{P}$ involving an adverse event (B) & 224 \\
\hline Number of individual cases involving event $E$ in relation to any other medicinal products but $P(C)$ & 117 \\
\hline Number of individual cases involving any other adverse events but $\mathrm{E}$ and any other medicinal products but $\mathrm{P}$ (D) & 27,242 \\
\hline Proportional Risk Ratio (PRR) calculated by the formula $[\mathrm{a} /(\mathrm{a}+\mathrm{b})] /[\mathrm{c} /(\mathrm{c}+\mathrm{d})]$ & 6.1001 \\
\hline Reporting Odds Ratio (ROR) calculated by the formula [a / c] / [b / d] & 6.2367 \\
\hline Relative Reporting Ratio (RRR) calculated by the formula $[a \times(a+b+c+d) /(a+c) \times(a+b)]$ & 5.8513 \\
\hline Expected & 1.0254 \\
\hline Chi-Square Test & 24.1334 \\
\hline Case Level Serious (CS) & 6 \\
\hline Event Level Serious (ES) & 6 \\
\hline Case Level Unexpected (CU) & 4 \\
\hline Event Level Unexpected (EU) & 4 \\
\hline
\end{tabular}


cerebral artery (MCA) with vascular occlusion. A bolus dose of intravenous (IV) alteplase $(5.9 \mathrm{mg}$ bolus plus infusion of $52.7 \mathrm{mg} /$ hour) was initiated 100 minutes after symptom onset. Due to a finding of major infarction in more than one half of the corresponding MCA territory, the alteplase infusion was withheld and she was transferred to another stroke unit for consideration of intraarterial mechanical clot extraction. Upon arrival, she was noted to have an audible wheeze and mild tachypnoea. Due to persistent occlusion of the right MCA second division, IV alteplase (59.4mg over 60 minutes) was recommended three hours following the initial bolus. After completing the alteplase infusion (4 hours after the initial bolus), she developed angioedema of the lips and tongue, with increased respiratory distress. The woman was treated with $200 \mathrm{mg}$ intravenous hydrocortisone, $50 \mathrm{mg}$ intravenous ranitidine, $25 \mathrm{mg}$ intravenous prochlorperazine, and $1 \mathrm{mg}$ intramuscular epinephrine (adrenaline). She was transferred to the ICU following intubation. Normotension and normal oxygenation was maintained during this period of anaphylaxis. Her serum tryptase levels were 2 and $4 \mathrm{Ug} / \mathrm{L}$ at the end of the alteplase infusion and 1.5 hours following the start of anaphylaxis, respectively. Eosinophil and basophil counts were not raised after the event. A CT brain scan, performed 24 hours after the alteplase infusion, revealed a small haemorrhage in the right internal capsule with mass effect and effacement of the right lateral ventricle. She was extubated the following day in the intensive care unit and the patient improved neurologically to a NIHSS of 18 , but required transfer to a nursing home upon eventual discharge. Action taken with the drug was unknown. There is a suggestion that concurrent use of an angiotensin converting enzyme (ACE) inhibitor and alteplase is associated with an increased risk of hemi-orolingual angioedema. In this case we are unable to conclude if the prior use of an ACE inhibitor increased the risk of anaphylaxis (Cheng et al., 2012). Allergic-type reactions have been reported with the use of alteplase including anaphylactoid reactions, laryngeal edema, orolingual angioedema, rash (unspecified), and urticaria. A cause and effect relationship to alteplase has not been established. Summary of individual cases were presented in table 1 .

\section{Analysis and interpretation of the reports}

All cases involved elderly patients. In case 1029 the suspect medication was alteplase, and as per the authors' comments it was not certain if the prior use of perindopril predisposed patient to the reaction. Two case reports involved patients with a medical history of anaphylactic reaction (for one of them, 1215, with the previous use of ramipril) and in one case (2269) of allergy to drugs, which are contributing risk factors. As per perindopril labels, the medication is contraindicated in patients with a history of hypersensitivity to any other ACE inhibitor, or of angioedema associated with previous ACE inhibitor therapy. In case 0302 it was not confirmed that the patient took perindopril. In addition, it was reported that the medications were used beyond their expiry date. Except for case number 1029 in which alteplase was suspected drug, reports do not contain detailed information especially about the symptoms of anaphylaxis. In case 2269 the patient with a history of drug reaction developed tongue swelling "suggesting an anaphylactic reaction". In Case 1044 the patient initially received $4 \mathrm{mg}$ dose for about 6 weeks and experienced anaphylaxis when the dose was increased to $8 \mathrm{mg}$. Anaphylaxis, anaphylactoid reaction, hypersensitivity, allergy, angioedema are events that have different MedDRA PTs (some even different HLTs), however, they are all under allergic conditions HLGT and some of their symptoms overlap. The exact definition for each of these events is not always used in adverse event reports and sometimes they might be used interchangeably. Although globally in the US or Europe the prescribing information do not specifically list anaphylactic reaction, they do list some signs and symptoms (e.g. angioedema of face, extremities, lips, mucous membranes, tongue, glottis and/or larynx, urticaria, pruritus, bronchospasm). In addition, they list anaphylactoid reaction and hypersensitivity. Anaphylactic reaction is listed in the Canadian Product labels under the section Less Common Clinical Trial Adverse Drug Reactions $(<1 \%)$, where adverse events irrespective of causal relationship to the drug, which occurred in $<1.0 \%$ of hypertensive and heart failure patients treated with perindopril in clinical trials, are listed.

\section{Summary of data}

Cases 1215, 2269 and 1044 showed that the patient had either a history of drug allergy or prior exposure to perindopril or ramipril. The occurrence of these events is suggestive of the possibility of previous sensitization and subsequent development of anaphylactic reaction following re-exposure. In cases 0302 it was not confirmed that the patient took perindopril and in case 1029 alteplase was the cause of the anaphylactic reaction.

\section{Statistical evaluation of data}

In order to determine the statistical significance of the anaphylactic reaction, the adverse drug event was processed through a model that calculated the Proportional Risk Ratio, Reporting Odds Ratio, Relative Reporting Ratio and Chi-Square for the event in relation to perindopril. The results are summarized in table 2 . The PRR, ROR, RRR and Chi-Square values further statistically confirms the potential for an anaphylactic reaction in patients treated with Perindopril.

\section{Evidence from the literature}

In a 12-month post-marketing surveillance study that included 47,351 hypertensive patients, anaphylaxis was reported as rare event. A study of anaphylactic reaction (anaphylaxis) among people who took perindopril erbumine, created by eHealthMe and based on 21 reports from FDA, revealed that out of 4,376 perindopril erbumine users that were studied, 21 people $(0.48 \%)$ had anaphylactic reaction (Speirs et al., 1998).

\section{Risk factors}

People with a personal or family history of any type of allergic reaction are at higher risk of developing anaphylactic reaction. Those with some co-morbid conditions (e.g. asthma, emphysema, chronic obstructive pulmonary disease or heart diseases) are at greater risk of developing complications during anaphylaxis.

\section{Late breaking information}

No significant information was received after the cut-off date of 04-Feb-2013.

\section{DISCUSSION}

Perindopril is an angiotensin converting enzyme (ACE) inhibitor indicated in the treatment of hypertension, symptomatic heart failure and reduction of risk of cardiac events in patients with a history of myocardial infarction 
and/or revascularization. Anaphylaxis is an acute, potentially life-threatening, IgE-mediated allergic reaction that occurs in previously sensitized people when they are re-exposed to the sensitizing antigen. Symptoms can include stridor, dyspnea, wheezing, and hypotension. Diagnosis is clinical. People with a personal or family history of any type of allergic reaction are at higher risk of developing anaphylactic reaction. Those with some comorbid conditions (e.g. asthma, emphysema, chronic obstructive pulmonary disease or heart diseases) are at greater risk of developing complications during anaphylaxis. There were five reports received by MAH to date where the patient experienced anaphylactic reaction with perindopril use. Of the five cases four were received from MHRA while one case was retrieved from the literature. In the literature case report (1029) the suspect product was alteplase, and the authors considered perindopril as the attributing factor in the occurrence of this event. In one case (0302) it was not confirmed if the patient was exposed to perindopril. In addition as per the reporter, the patient took expired medications. For the remaining three cases, (1215, 2269 and 1044) although reporters did not provide the complete information, no alternative explanation for the event could be provided. The history of allergy or prior exposure to perindopril or ramipril in these cases is suggestive of the possibility of previous sensitization and subsequent development of anaphylactic reaction following re-exposure. A study of anaphylactic reaction (anaphylaxis) among people who took perindopril erbumine created by eHealthMe and based on 21 reports from FDA revealed that out of 4,376 perindopril erbumine users that were studied, 21 people $(0.48 \%)$ had anaphylactic reaction. There were approximately 513.93 million patient days of exposure to firms perindopril since commercialization. Allergic type reactions are described in firms and innovator SPCs, however, anaphylactic reaction is not specifically listed.

\section{CONCLUSION}

Anaphylactic reaction as a specific term is only listed in the Canadian Product Monograph under the section Less Common Clinical Trial Adverse Drug Reactions $(<1 \%)$, where adverse events irrespective of causal relationship to the drug, which occurred in $<1.0 \%$ of hypertensive and heart failure patients treated with perindopril in clinical trials, are listed. Three out of five case reports that were entered in the drug safety database suggestive of a possible association between perindopril and anaphylactic reaction.

\section{REFERENCES}

Cheng JN, Lee A, Jannes J, Heddle RJ, Koblar SA. (2012). A patient with anaphylaxis after alteplase infusion. Journal of Clinical Neuroscience 19(2): 314-315. [DOI]

Jain PS, Badreshiya PR, Chalikwar SS, Todarwal AA, Surana SJ. (2012). Validation of a dissolution method with RP-HPLC analysis for perindopril erbumine and indapamide combination tablet. Chemical Industry \& Chemical Engineering Quarterly 18 (1): 19-25. [DOI]

Joseph J, Philip B, Sundarapandian M. (2011). Method development and validation for simultaneous estimation of perindopril erbumine and indapamide by RP-HPLC in pharmaceutical dosage forms. International Journal of Pharmacy and Pharmaceutical Sciences 3(4): 288-293.

Nagalakshmi C, Rani AV, Bhawani S, Sekaran CB. (2012). Determination of angiotensin-converting enzyme inhibitor, perindopril erbumine, in bulk and tablet dosage form with HPLC. American Chemical Science Journal 2(4): 161-176.

Patil Pradeep S, More Akshata K., Kadam Sunil, Vishwasrao Vishal, Patel Yagnik. (2013). Formulation and evaluation of orodispersible tablets of Perindopril erbumine using natural superdisintegrant. Journal of Drug Delivery \& Therapeutics 3(5): 44-48.

Prajapati JP, Patel MB, Prajapati RJ, Prajapati NA. (2011). Simultaneous determination of perindopril erbumine and amlodipine besylate by absorption factor method. International Journal of Applied Biology and Pharmaceutical Technology 2(3): 230-233.

Ratnaparkhi MP. (2012). Formulation and development of taste masked orally disintegrating tablets of perindopril erbumine by direct compression method. Pharmaceutica Analytica Acta 3:162. [DOI]

Riyaz SMD, Vasanth PM, Ramesh M, Ramesh R, Ramesh T. (2012). A sensitive RP-HPLC method development and validation for the simultaneous estimation of perindopril erbumine and amlodipine besilate in tablet dosage form. International Journal of Chemical and Life sciences 1 (2): 1033-1038.

Sharma S, Sharma MC. (2011). UV-Spectrophotometric method for the perindopril erbumine in pharmaceutical formulations using indigo carmine. American-Eurasian Journal of Scientific Research 6 (4): 210 216.

Speirs C, Wagniart F, Poggi L. (1998). Perindopril post marketing surveillance: a 12 month study in 47,351 hypertensive patients. British Journal of Clinical Pharmacology 46(1): 63-70. [DOI]

Sridevi N, Jahnavi G, Sekaran CB. (2012). Spectrophotometric analysis of Perindopril Erbumine in bulk and tablets using bromophenol blue. Der Pharmacia Lettre 4(1):159-169.

Tarkase KN, Mahajan PB, Suruse SD. (2013). Simultaneous estimation of perindopril erbumine and in dapamide in combined dosage form using derivative spectrophotometric method. International Journal of Pharma Research \& Review 2(7): 7-11.

Varma D, Lakshmana Rao A, Dinda SC. (2013). Validated stability indicating reverse phase HPLC method for the simultan eous estimation of perindopril and indapamide in pharmaceutical dosage forms. International Journal of Pharmacy 3(1): 277-289. 